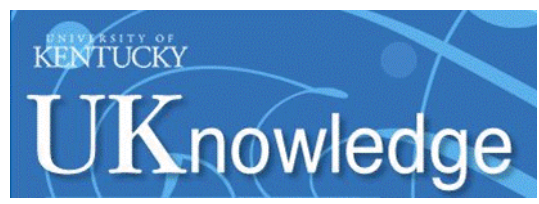

University of Kentucky

UKnowledge

\title{
Coffee: A Selected Overview of Beneficial or Harmful Effects on the Cardiovascular System?
}

Thomas F. Whayne Jr.

University of Kentucky, thomaswhayne@uky.edu

Follow this and additional works at: https://uknowledge.uky.edu/internalmedicine_facpub

Part of the Cardiology Commons

Right click to open a feedback form in a new tab to let us know how this document benefits you.

\section{Repository Citation}

Whayne, Thomas F. Jr., "Coffee: A Selected Overview of Beneficial or Harmful Effects on the Cardiovascular System?" (2015). Internal Medicine Faculty Publications. 59.

https://uknowledge.uky.edu/internalmedicine_facpub/59

This Article is brought to you for free and open access by the Internal Medicine at UKnowledge. It has been accepted for inclusion in Internal Medicine Faculty Publications by an authorized administrator of UKnowledge. For more information, please contact UKnowledge@lsv.uky.edu. 
Coffee: A Selected Overview of Beneficial or Harmful Effects on the Cardiovascular System?

Digital Object Identifier (DOI)

https://doi.org/10.2174/1570161112666141002121429

Notes/Citation Information

Published in Current Vascular Pharmacology, v. 13, issue 5, p. 637-648.

The copyright holder has granted the permission for posting the article here.

The document available for download is the authors' post-peer-review final draft of the article.

This article is available at UKnowledge: https://uknowledge.uky.edu/internalmedicine_facpub/59 


\title{
Coffee: A Selected Overview of Beneficial or Harmful Effects on the
} Cardiovascular System?

\author{
Thomas F. Whayne, Jr, MD, PhD \\ Professor of Medicine (Cardiology) \\ Division of Cardiovascular Medicine \\ Gill Heart Institute \\ University of Kentucky \\ Lexington, Kentucky, USA
}

\section{Corresponding author:}

Thomas F. Whayne, Jr, MD, PhD

Gill Heart Institute

326 Wethington Building

900 South Limestone Street

Lexington KY 40356-0200, USA

Tel: $\quad$ (859) 323-

FAX: (859)

E-mail: $\quad$ twhayn0@uky.edu $\quad(0$ is the number zero) 


\begin{abstract}
With a history that began in 800 A.D., coffee is the most popular drink known and as a result, the issues regarding its physiologic effects deserve attention. Maintaining alertness is a well-known benefit and in addition, the cardiovascular (CV) effects of the active compounds, which include polyphenols and caffeine, must be considered. Genetics are relevant and where slow caffeine metabolism is inherent, the risk of nonfatal myocardial (MI) has been shown to be increased. Overall risk for coronary heart disease (CHD) is not supported and unless there is excessive intake, congestive heart failure (CHF) is not adversely affected; in moderation, there may be some benefit for CHF. There is no apparent increased risk of sudden cardiac death (SCD). Overall, there also appears to be a beneficial inverse association with all-cause mortality, although this is not absolute for extra heavy intake. Benefit in reducing stroke also has supportive evidence. Hypertension is not increased by coffee. Boiled and unfiltered coffee appears to increase plasma cholesterol and triglycerides but for the overall metabolic syndrome, there appears to be benefit. There is also some evidence that paper-filtered coffee results in an increase in some markers of inflammation. Association of coffee with arrhythmias has been a major concern though in moderation it is not a significant overall problem. Therefore, only if a patient were to associate major arrhythmic symptoms with coffee would cessation have to be advised. Where coffee clearly shines from a CV standpoint is in the established decrease in onset of type 2 diabetes mellitus (DM). Any benefit or harm has always been attributed to caffeine as the apparent major component. However, coffee contains a myriad of compounds, including polyphenols. These other substances may be most relevant for potential benefit or harm and some of these may be partially removed or altered by coffee preparation methods such as paper filtration. Multiple studies support this by what appears to be no CV advantage or disadvantage for decaffeinated coffee. The bottom line on coffee, for those who enjoy the brew, is that it is a wonderful beverage with rare associated $\mathrm{CV}$ disadvantage and with much to recommend it from an overall CV standpoint.
\end{abstract}

Key words: Cardiovascular, coffee, coronary heart disease, congestive heart failure, diabetes mellitus, myocardial infarction, sudden cardiac death. 
Abbreviations:

ABI: $\quad$ Ankle-brachial index

AF: $\quad$ Atrial fibrillation

ApoA-1: $\quad$ Apolipoprotein A-1

ApoB: $\quad$ Apolipoprotein B

BP: $\quad$ Blood pressure

CGAs: Chlorogenic acids

CHD: $\quad$ Coronary heart disease

CHF: Congestive heart failure

CI: Confidence interval

CQAs: $\quad$ Caffeoylquinic acids

CV: $\quad$ Cardiovascular

CYP1A2: $\quad$ Cytochrome P450 1A2

DM: $\quad$ Diabetes mellitus

FMD: $\quad$ Flow-mediated dilatation

HDL-C: High-density lipoprotein cholesterol

LDL: Low-density lipoprotein

LDL-C: Low-density lipoprotein cholesterol

MetS: $\quad$ Metabolic syndrome

MI: Myocardial infarction

NAFLD: $\quad$ Non-alcoholic fatty liver disease

OR: $\quad$ Odds ratio

PAD: $\quad$ Peripheral arterial disease

RR: $\quad$ Relative risk

SCD: $\quad$ Sudden cardiac death 


\section{Introduction}

The history of coffee begins in 800 years A.D. in the Ethiopian highlands with the legend of Kaldi the goat herder.[1] While caring for his herd, Kaldi noted that after some of the goats ingested grains of a specific plant (that turned out to be coffee), they became more restless and playful. After sharing his observations with the abbot of a local monastery, Kaldi was requested to prepare a drink from the grains, and he subsequently shared it with the monks in the monastery. Thus, coffee was established as a desirable beverage and its use spread rapidly in the African locale, to the Arabian peninsula, and on to Europe.[2] The purpose of this article is to review the cardiovascular $(\mathrm{CV})$ effects of the most popular drink in the world (Table 1).

\section{Brief summary of possible beneficial physiologic effects}

Well accepted physiologic effects of coffee are its ability to help avoid somnolence and maintain alertness. The bioactive compounds in coffee appear to be chlorogenic acids (CGAs) which are polyphenols, caffeine, the pentacyclic diterpenes known as cafestol and kahweol, trigonelline, and melanoidins.[3] The CGAs consist of the caffeoylquinic acids (CQAs), especially 5-CQA, as well as lower amounts of feruloylquinic acids and dicaffeoylquinic acids. CQAs have an antioxidant effect but following ingestion of coffee, unmetabolized CQAs attain only a transient and low peak plasma concentration and cannot compete with more potent antioxidants in the body. It appears that a direct antioxidant effect for CGAs from coffee has been discarded. However, CGAs may have an antithrombotic effect by inhibiting platelet activity.[4] Both caffeic acid and 5-CQA from coffee have been shown to have antiplatelet activity with mice platelets in vitro and in vivo.[5] This appears to occur in association with suppressed expression of P-selectin, a biomarker for platelet activation. An anti-inflammatory effect of coffee may also be a beneficial effect. Dihydrocaffeic acid has been shown in human keratinocyte cells to have a down regulation of ultraviolet light-induced production of the proinflammatory cytokine interleukin-8.[6] Suggesting two other potentially beneficial effects, it has been shown that green coffee extract and 5-CQA given orally to hypertensive rats produced potentially beneficial alterations in blood pressure (BP) and endothelial function.[7] An effect on the latter was suggested by attenuation of the depressor effect from concurrent injection of atropine sulfate, mediated by the muscarinic acetylcholine receptors.

Caffeine is a major component of coffee and a major reason for its ingestion but for it or any bioactive component to benefit $\mathrm{CV}$ disease, it would have to improve major $\mathrm{CV}$ risk factors such as lipids or BP, or else have an in vivo antioxidant effect at physiological levels.[4] There 
appears to be no major suggestion of a beneficial lipid effect of caffeine unless, by contributing to weight loss, it could indirectly decrease plasma lipids. In addition, caffeine itself has no lowdensity lipoprotein (LDL) antioxidant effect though some caffeine metabolites such as 1methylxanthine and 1-methyluric acid are effective in preventing LDL oxidation.[8] The major polyphenol in coffee is CGA, which is an ester of caffeic acid and quinic acid. CGA is an in vitro antioxidant with 2 phenolic groups for radical scavenging by proton transfer. The apparent antioxidant content of coffee is $1.249 \mathrm{mmol} / 100 \mathrm{~g}$.[9] It has been calculated that a $180 \mathrm{ml}$ cup of brewed coffee provides $396 \mathrm{mg}$ of polyphenols. [4] When this data is combined with per capita consumption of coffee, it appears that coffee is the largest source of antioxidants in the United States diet, potentially of significance for possible CV benefit.

\section{Genetics}

The metabolism of caffeine contained in coffee occurs via the polymorphic cytochrome P450, 1A2 (CYP1A2) enzyme as the principal coffee metabolizing enzyme;[10-12] individuals homozygous for the CYP1A2*1A allele metabolize caffeine rapidly.[10] On the other hand, carriers of the variant CYP1A2*1F allele show a much slower caffeine metabolism. Cornelis et al. studied whether a specific CYP1A2 genotype changes the risk for acute myocardial infarction (MI).[10] These authors studied 2,014 cases of first acute nonfatal MI versus 2,014 controls, with both groups living in Costa Rica between 1994 and 2004, that were matched for age (median age $59)$, sex, and area of residence. A total of $55 \%$ of cases $(n=1,114)$ and $54 \%$ of controls $(n=1,082)$ were carriers of the slow caffeine metabolism $* 1 \mathrm{~F}$ allele. With the slow $* 1 \mathrm{~F}$ allele carriers, the multivariate-adjusted odds ratios (ORs) and $95 \%$ confidence intervals (CIs) of nonfatal MI associated with the consumption of less than 1, 1, 2-3, and 4 or more cups of coffee per day were $1.00,0.99$ (0.69-1.44), 1.36 (1.01-1.83), and 1.64 (1.14-2.34), respectively.[10] On the other hand, the ORs $(95 \% \mathrm{CIs})$ for the rapid metabolizing *1A/*1A genotype were $1.00,0.75(0.51$ 1.12), 0.78 (0.56-1.09, and 0.99 (0.66-1.48). For the gene-coffee interaction, $\mathrm{P}=0.04$. Therefore, coffee consumption was associated with increased nonfatal MI risk only among individuals with slow caffeine metabolism in their study.[10]

\section{Sudden death}

Bertoia et al. studied the possible role of alcohol and caffeine intakes in the development of sudden cardiac death (SCD) as studied in 93,676 postmenopausal women participating in the Women's Health Initiative Observational Study, enrolled between 1993 and 1998, and followed until August 2009.[13] They found that one drink of alcohol per day (5.1-15 g/d) may have an association with a decreased SCD risk in this population of women but that the association had statistical significance for a model using the most recent alcohol intake. For coffee, the authors

found no association between SCD and total caffeine intake in $\mathrm{mg} / \mathrm{d}$ or total cups of caffeinated coffee, decaffeinated coffee. Also, no SCD association for caffeinated tea was found. 


\section{Arrhythmias}

A major consideration for recommending a decrease in coffee and caffeine consumption is whenever there is a significant association with symptomatic arrhythmias, despite the absence of any association with increased SCD. Park and Grubb reported on a 38 year-old male patient with nausea, rapid palpitations, and presyncope, which occurred in association with the consumption of a large amount of strong French coffee.[14] His electrocardiogram showed a tachycardia with a slightly increased QRS duration of right bundle branch block pattern with left axis deviation and supraventricular tachycardia was initially diagnosed. With a subsequent electrophysiologic study, he was found to have a fascicular tachycardia as a rare presentation of ventricular tachycardia. This was successfully treated with radiofrequency catheter ablation.

Any relationship of the most prevalent sustained arrhythmia, atrial fibrillation (AF), to coffee and caffeine appears to be supported only by heterogeneous data in the available literature as evaluated by Caldeira et al.[15] These authors assessed the association between chronic exposure to caffeine and AF. The following were used as data sources up to and including December 2012: PubMed, CENTRAL, ISI Web of Knowledge and LILACS. They used 7 observational studies including 115,993 individuals with 6 cohorts and one case-control study. Caffeine was not related to increased AF risk (OR 0.92, 95\% CI 0.82 to 1.04). For low-dose caffeine, it even appeared that there might be a protective effect to decrease AF risk (OR 0.85, 95\% CI 0.78 to 0.92). In addition, exposure to caffeine that was related exclusively to drinking coffee had no influence on AF. In an editorial analysis of the publication of Caldeira et al.,[15] Bhave and Hoffmayer[16] concluded that the available data supported that low to moderate consumption of caffeine is safe in regard to not causing AF, that caffeine in moderation does not appear to promote cardiac dysrhythmias; nevertheless, there did not appear to be sufficient data to establish coffee as protective.[16]

\section{Diabetes mellitus (DM)}

Publications on possible health effects of coffee have undergone extensive study. Multiple population studies have assessed the relationship of coffee drinking and type 2 DM and have concluded that frequent coffee drinking appears to decrease the risk of type 2 DM.[17] However, data regarding habitual coffee consumption and type $2 \mathrm{DM}$ risk are very recent as reviewed by Muley et al.[18] These authors studied 13 cohort studies that included 47,387 participants, and 9,473 incident cases of type 2 DM. The risk of DM was assessed with different degrees of coffee consumption. It was concluded that regular consumption of coffee is associated with a decreased risk of type 2 DM. The participants that drank 4 to 6 cups and those that drank over 6 to 7 cups of coffee each day had a lesser type 2 DM risk than those participants who drank less than 2 cups per day. There appeared to be an advantage of filtered coffee over pot-boiled coffee. Muley et al. actually reported an advantage of decaffeinated coffee over caffeinated 
coffee.[18] Also, there was a stronger inverse correlation for type $2 \mathrm{DM}$ in the age group under 60 years. Nevertheless, these authors did not advocate the recommendation to increase the consumption of coffee as a public health policy, especially without widespread assessment of postprandial hyperglycemia and insulin sensitivity.[18] Jiang et al. studied the relationship of coffee and caffeine to type $2 \mathrm{DM}$ patients by identification of studies they considered pertinent from a search of PubMed and EMBASE.[19] These authors performed a dose-response assessment showing that the occurrence of type 2 DM decreased by $12 \%$ (Relative Risk [RR] 0.89, CI 0.82) when coffee intake was increased by 2 cups/day.[19] The decrease with decaffeinated coffee was almost as great and was found to be $11 \%$ (RR 0.86, CI 0.82-0.91) per every 2 cup/day increase. With caffeine intake, there was a $14 \%$ decrease in type 2 DM per every increase in caffeine of $200 \mathrm{mg} /$ day (RR 0.86, CI 0.82-0.91).[19] Other analyses also support the benefit of coffee to decrease type 2 DM risk in response to dose.[20] Data from the Nurses' Health Study (NHS) also support the benefit of decreased occurrence of type 2 DM with a combined assessment of caffeinated/decaffeinated coffee (RR 8\%); the Health Professionals Follow-Up Study (HPFS) showed separate benefit with both caffeinated and decaffeinated coffee (RR 4\% and 7\%, respectively).[21] The decrease in type 1 DM was significant in the case of both the NHS and HPFS studies.

Natella and Scaccini extensively reviewed the part played by coffee regarding the risk of DM.[22] Regarding a mechanism for the effect of coffee, they commented on the status of coffee as a complicated mixture of multiple substances that include, of course, caffeine, as well as phenols, niacin, fiber and minerals. These authors discussed that despite the good evidence for coffee in protecting against type $2 \mathrm{DM}$, there must be awareness of opposing reported results that describe an adverse effect of caffeine on glucose metabolism even with reports of associated hyperglycemia and decreased insulin sensitivity associated with caffeine.[22] They interpret their analysis as showing that these negative effects of caffeine are favorably altered when caffeine is present within coffee as a complex conglomeration of various substances and that regular coffee consumption has a protective benefit in decreasing the onset of type $2 \mathrm{DM}$. The authors considered that potential mechanisms to decrease type $2 \mathrm{DM}$ associated with coffee, such as the presence of antioxidant effects (e.g. phenols) and anti-inflammatory effects (e.g. phenols) are considerations but these are without definitive proof. Therefore, there is still insufficient evidence to specifically recommend increased coffee consumption to prevent type $2 \mathrm{DM}$, despite the reported decrease in incidence. The situation regarding any benefit of coffee consumption for type 1 DM and gestational DM has definitely not been clarified.[22]

\section{Dietary considerations}

Coffee is, or course, a major source of caffeine and caffeine can be considered a major dietary component due to its widespread use. Wierzejska, in an article in Polish, commented that women around Warsaw had an average daily caffeine consumption of $251 \mathrm{mg}$ with $15 \%$ consuming in excess.[23] It currently appears that a dose of $400 \mathrm{mg}$ of caffeine per day is not 
associated with negative effects. The author states that there is an average daily consumption of caffeine in Europe that ranges from 280 to $490 \mathrm{mg}$ with the highest consumption in Scandinavia due to a very high level of ingestion of coffee. There is a general recommendation that pregnant women eliminate coffee and caffeine from their diet and Wierzejska described caffeine metabolism in pregnancy as decreased also with free passage of caffeine and its metabolites across the placenta.[23] The average mean daily intake of caffeine in the United States has been reported by Barone and Roberts as $4 \mathrm{mg} / \mathrm{kg}$ with youth under age 18 consuming a mean daily amount of $1 \mathrm{mg} / \mathrm{kg}$. Ground roasted coffee in an amount of $5 \mathrm{oz}$ has $85 \mathrm{mg}$ caffeine with $18 \mathrm{mg}$ caffeine in a $6 \mathrm{oz}$ cola.[24] For the supposedly average $80 \mathrm{~kg}$ man, this calculates out at $320 \mathrm{mg}$ and it would appear that coffee is the major source although many groups concentrate on colas and "energy" drinks. To assess the above caffeine statistics, available statistics from 1999 showed that the average daily consumption of coffee in the United States was 3.1 cups per day among coffee drinkers.[25] On a per capita basis in the United States, the approximate intake for men is 1.9 cups of coffee per day; for women, it is 1.4 cups of coffee per day.

\section{Cardiovascular (CV) risk}

\section{General considerations}

A major purpose of this article is to assess available data on coffee and CV risk. Ding et al. performed an extensive and recent meta-analysis of coffee consumption over a long term.[26] They interpreted their meta-analysis as suggesting a nonlinear relationship between CV risk and the consumption of coffee. Their data analysis supported that moderate consumption of coffee had an association with decreased CV risk. They found that the lowest CV risk occurred with 3-5 cups of coffee per day, but exceeding that with what could be considered heavy coffee consumption, there was no associated increase in CV risk.[26] Multiple other extensive analyses have assessed CV risk. However, as discussed by O'Keefe et al., most information on the medical ramifications of coffee have utilized observational information and unfortunately, there is only a minimal number of randomized controlled trials. Therefore, the presence of only an association fails to prove causation.[27] Nevertheless, coffee is the major source of caffeine in the United States and there is a daily consumption of coffee by in excess of 150 million people there.[28] There is increasing information that individuals who drink coffee as compared to those who do not, may be less likely to go on in the future to have type $2 \mathrm{DM}$, stroke, depression, death from any cause, as well being less likely to develop neurodegenerative diseases, which include Parkinson's disease and Alzheimer's disease. Especially when coffee is taken without added cream, various syrups, or sugar, as a calorie-free drink, it offers a greater probability of health benefit, especially if there are no perceived adverse subjective effects.[28] Floegel et al. assessed data from the European Prospective Investigation into Cancer and Nutrition (EPIC)-Germany study involving 42,659 participants.[29] Their analysis found that consumption of coffee of 4 or more cups of coffee per day, caffeinated or decaffeinated, compared to less than one cup per day, 
was not associated with any significant change in risk for MI, stroke, or cancer, but the increased coffee consumption was linked to a decreased type 2 DM risk.

Montagnana et al. looked at the results of multiple epidemiological studies that suggested a U-shaped relationship associated with the consumption of coffee and the occurrence of $\mathrm{CV}$ events, including mortality.[30] These authors concluded that lower CV risk was associated with low coffee consumption of less than one cup per day or associated with high consumption of coffee of 4 or more cups of coffee per day. Surprisingly, an intermediate consumption of 2-4 cups of coffee per day demonstrated higher CV risk. They suggested an explanation attributable to the different substances in coffee such as CGAs (which are polyphenols), possibly effective in decreasing CV risk by decreasing the aggregation of platelets, the incidence of type $2 \mathrm{DM}$, the occurrence of inflammation, and the incidence of hypertension. They associated a possible relationship of caffeine to decreased $\mathrm{CV}$ risk due to weight reduction, increased fibrinolysis, and increased arterial flow-mediated dilatation (FMD).[30]

\section{Method of preparation of coffee}

How coffee is prepared may also be an issue as pointed out by Lopez-Garcia. Coffee prepared by a nonfiltered method such as boiled, French press, and espresso, does not remove diterpenes and has been shown to increase plasma total cholesterol and low-density lipoprotein cholesterol (LDL-C).[31] This adds some confusion in that nonfiltered coffee could contribute to increased CV risk, so it is important to review this issue. Ahola et al. studied 20 healthy volunteers and their consumption of strong boiled coffee and the same boiled coffee but passed through filter paper over 4 week periods, and crossed over for the volunteers.[32] These authors found that when consuming boiled unfiltered coffee, serum total cholesterol, LDL-C, triglycerides, and apolipoprotein B (apoB) levels were significantly higher than during the period of ingestion of the same, but filtered, coffee. High-density lipoprotein cholesterol (HDL-C) and apolipoprotein A-1 (apoA-1) levels were not altered by the preparation method in this study. They considered that whatever hypercholesterolemic factor is present in boiled coffee is, most likely, lipid soluble and absorbed by the paper filters. Van Dusseldorp et al. studied 64 healthy volunteers and found that total cholesterol, LDL-C, and apoB levels were higher in their group ingesting boiled but unfiltered coffee. HDL-C and apoA-1 as well as triglyceride levels did not differ significantly.[33] They found that serum campesterol level, which reflects cholesterol absorption, stayed constant whereas serum lathosterol, as a measure of cholesterol synthesis, increased $11 \%(\mathrm{p}<0.05)$. The lathosterol/cholesterol ratio, however, remained the same. It appeared to van Dusseldorp et al. that removal of whatever hypercholesterolemic factor is present in boiled nonfiltered coffee, occurs by the retention of lipids during filtration.[33] Increased cholesterol biosynthesis or absorption when consuming boiled nonfiltered coffee did not appear to them to be a mechanism. Multiple other studies support an increase in total cholesterol, LDL-C, and apoB from the ingestion of boiled coffee.[34-37] Aro et al. also found a direct increase in total cholesterol, LDL-C, and apoB, as related to the amount of boiled coffee consumed.[36] On the other hand, boiled coffee does not appear to affect the actual protein and 
lipid composition of the LDL-C.[37] Rosmarin et al. studied filtered coffee alone in a moderate dose of $3.6 \mathrm{cups} / \mathrm{d}$ and their results supported the lack of any effect of filtered coffee on total cholesterol, LDL-C, apoB, and HDL-C after controlling for diet, use of coffee creamer, and cigarette smoking.[38] In a study of Israeli factory workers, Green and Harari obtained detailed information on the ingestion of coffee and tea and lipids in 3,838 Israeli men and 1,511 Israeli women.[39] They found an especially significant and positive association of increased total cholesterol and LDL-C with boiled coffee. However, other results are not consistent as in the case of the study of filtered coffee by Fried et al. which reported that ingestion of $720 \mathrm{~mL} / \mathrm{d}$ of caffeinated filtered coffee resulted in a statistically significant increase in plasma total cholesterol, which appeared to result from LDL-C and HDL-C increases.[40] Their results are not consistent with the mainstream regarding no LDL-C increase associated with filtered coffee and further specific clarification is needed. Results from an Italian study of 8,983 subjects reported that 1-3 cups/d resulted in an adjusted increase in serum total cholesterol of $3.4 \mathrm{mg} / \mathrm{dL}$ $(0.088 \mathrm{mmol} / \mathrm{L})$ with an increase up to $9.6 \mathrm{mg} / \mathrm{dL}(0.248 \mathrm{mmol} / \mathrm{L})$ in those drinking over 5 cups/d.[41] In the Italian method, ground coffee is preheated with steam and water goes through the ground coffee at a higher temperature compared with other techniques of brewing.[41] These results appear consistent with the higher lipid levels reported with boiled nonfiltered coffee. However, inconsistent observations have been noted, as happened with the small study of D'Amicis et al. of 84 men in which the Italian method of brewing coffee showed no significant differences in total cholesterol, LDL-C, and HDL-C.[42]

Two coffee diterpines, cafestol and kahweol, present in unfiltered coffee, appear to be significant factors accounting for increased LDL-C [43, 44] as well as increased very lowdensity lipoprotein cholesterol with also a slight reduction in HDL-C, according to van Tol et al.[43] They observed some increased activity of proteins in serum involved in transfer of lipids and suggested such an increase as a possible mechanism associated with the increased lipid levels with boiled coffee. De Roos specifically studied the effect of French-press coffee (a nonfiltered method with only a strainer to catch coffee grounds) in 46 subjects and found an associated persistent increase in cholesteryl ester transfer protein (CETP).[45] They suggested this as a potential contributing mechanism for the LDL-C increase in boiled, unfiltered coffee resulting from cafestol and kahweol. Another potential mechanism was studied by Rustan et al in HepG2 cells.[46] These authors found that cafestol and kahweol decreased the hepatic LDL-C receptors, which could be an explanation of increased LDL-C associated with these coffeerelated diterpines in boiled, nonfiltered coffee.

Phenolic acids with antioxidant activity may be a significant beneficial factor in coffee. In a study of 10 healthy volunteers who drank $200 \mathrm{~mL}$ of filtered coffee after an overnight fast, Natella et al. found increased resistance of LDL-C to oxidative modification.[47] They attributed this to the new presence of phenolic acids from coffee in LDL-C. Coffee is also a source of polyphenols which have also been shown to have antioxidant activity as well as decreasing vascular inflammation.[48] Both of these effects could offer significant benefit in reducing CV risk. 


\section{Coffee epidemiology and plasma lipids}

Although much of the effect of coffee on plasma lipids can be explained by the method of preparation, overall epidemiology has to be considered and multiple studies show an increase in especially LDL-C and then associate this with increased CV risk. However, much confusion occurs in that there are also studies that show no increase in plasma lipids with caffeinated coffee. Williams et al. studied a small group of 77 men and found total cholesterol and LDL-C were ultimately related to the amount of coffee consumed.[49] These authors found that LDL-C and apoB were not related to a coffee intake of up to 2 cups/d but rather, were associated with a coffee ingestion of greater than 2-3 cups/d. On the other hand, Aro et al., in a brief study, assessed 12 healthy volunteers aged 33-45 and had the subjects consume, successively, for 3week intervals, 8 cups/d of instant caffeinated coffee (total caffeine $520 \mathrm{mg}$ ), instant tea (total caffeine $200 \mathrm{mg}$ ), or rose hip tea (no caffeine).[50] There were no resultant differences in total cholesterol, LDL-C, HDL-C, triglycerides and the authors concluded that caffeinated coffee has no detectable effect on these lipids in healthy individuals with normal lipids. The Beaver County study of 472 white men and women in Beaver County, Pennsylvania in 1981-1982 was reported by Donahue et al., and it was found that no coffee, coffee intake of 1-2 cups/d, and coffee intake of 3 or more cups/d, resulted in no effect on total cholesterol, LDL-C, HDL-C, apoB, or apoA-1 in men or women.[51] The only significant lipid effect found was that women drinking 3 or more cups/d had significantly lower triglyceride concentrations $(\mathrm{P}<0.05)$, a finding of indeterminate $\mathrm{CV}$ significance. Paoletti et al. also reported that chronic coffee drinking did not affect total cholesterol, HDL-C, apoB, or apoA-1 in a group of healthy subjects.[52] This observation also appeared to hold for individuals with a high consumption of coffee. The extensive and famous Framingham Study also failed to resolve any relationship of coffee to CV disease. As reported by Wilson et al. in a combined analyses of 2,648 men with 549 cases of CV disease and 3,566 women with 462 cases of $\mathrm{CV}$ disease, no association of CV disease and ingestion of coffee was found in smokers, nonsmokers, men, or women.[53] In this Framingham substudy, men had a significant negative association of coffee and total cholesterol whereas women had a positive association of coffee and LDL-C. Framingham appears to offer no clarification to the lipid issue but supports the absence of an increase in CV disease associated with caffeinated coffee.[53] To add further confusion to differences in various groups, Kohlmeier et al. studied the association of coffee consumption and lipids in 395 young adults randomly selected, and in 385 elderly adults, in Germany.[54] After adjusting for various confounding factors, they found statistically significant total cholesterol and LDL-C increases only in the group of younger men, which was not noted in their elderly subjects. Carson et al. also studied such an age relationship in a group of 1,035 elderly white women ranging in age from 65 to 90 and, after adjustments for interfering factors, found no significant association of total cholesterol, LDL-C, and HDL-C with caffeinated coffee.[55]

Jee et al. performed a meta-analysis using a Medline search of English-language literature before December 1998, discussions with experts, and a bibliographic review that 
resulted in the identification of 14 coffee-intake trials.[56] The result of their meta-analysis was an observation of a direct response relationship between coffee intake and total cholesterol as well as LDL-C $(\mathrm{P}<0.01)$. They found increases in the lipids studied in subjects with hyperlipidemia already present and in the trials involving boiled coffee.[56] In the trials with filtered coffee, there was only a small increase in total cholesterol, offering further support of filtration removal of lipid-augmenting substances. In another meta-analysis in 1993, Kokjohn et al. searched Medline back to 1965 with the requirement that articles utilized had to have involved original results, have reported serum total cholesterol, and have been peer-reviewed for publication.[57] They found that there was a positive association of increased total cholesterol with increased coffee drinking with the differences assessed for drinking 4 or more cups of coffee/d, less than 1 cup of coffee/d, or no coffee. Again, there was an increased relationship of boiled coffee to total cholesterol as compared to filtered, decaffeinated, or instant coffee. Consistent with this, Cai et al. searched several English and Chinese electronic databases, including 1,017 subjects, and found that coffee consumption and especially unfiltered coffee consumption resulted in increases in total cholesterol, LDL-C, and triglycerides, with the increases related to the level of coffee consumption.[58]

Diet has been considered to be a possible confounding effect to explain any association of increased coffee consumption with increased lipids. In assessing this possibility, Haffner et al. looked at any relationship between serum lipid levels and the drinking of caffeinated coffee and any other caffeinated drinks.[59] Their study was population-based and involved 1,228 women and 923 men. They found a positive association between the consumption of coffee versus total cholesterol and LDL-C, after taking into account age, alcohol, race, obesity, and tobacco use. Since Haffner et al. found no association of lipids with tea or colas, it suggested to them that their observation was not just due to caffeine.[59] As a result, they evaluated the possibility of a more atherogenic diet in association with increased use of coffee. In men, there was increased caloric intake from total fat, saturated fat, and cholesterol associated with increased use of coffee. However, this association did not hold in women in the study but nevertheless, consideration of the effect of diet appears valid but unresolved. Another dietary observation and caveat with coffee were pointed out by Cheung et al., who studied the effect of a single cup of coffee in 40 individuals, $60 \mathrm{~min}$ before drawing blood for analysis.[60] Their results showed that this one cup of coffee ingested an hour before phlebotomy caused a statistically significant, although minimal, increase in total cholesterol and HDL-C with black coffee, and in triglycerides when cream and sugar were added.

The relationship of coffee drinking and lipids as affected by smoking has also been assessed. Jossa et al. reported on the Olivetti Heart Study of 856 coffee drinkers.[61] They performed adjustments for possible confounding factors. They found a statistically significant positive trend between coffee drinking and total cholesterol only in smokers, which suggested to the investigators that smoking status is key for any total cholesterol increase by coffee drinking. Such effects and differences show how difficult it is to clarify the association of coffee and lipids. 
The relationship of caffeine or other components of coffee also have to be considered regarding plasma lipids. Superko et al. studied 181 men who consumed caffeinated coffee for 2 months as the control and then were randomized to decaffeinated coffee, or no coffee, for 2 months.[62] They found that the switch from caffeinated to decaffeinated coffee actually resulted in increased LDL-C and apoB, whereas there was no increase in these lipid components in the no-coffee group. This, of course, suggested that instead of caffeine, another substance (or substances) in coffee might be the causative factor for increased LDL-C and apoB. A similar result regarding decaffeinated coffee was reported by van Dusseldorp et al., who studied 23 women and 22 men aged 25-45 years who were drinking 4-6 cups of caffeinated coffee per day.[63] A randomized trial was set up for the participants to drink 5 cups of caffeinated coffee/d for a total of 6 weeks, then decaffeinated coffee for 6 weeks, or participate in reverse. It was found that the effect on total cholesterol, HDL-C, and triglycerides was essentially null and the authors concluded that going to decaffeinated coffee had no effect on lipids. Thompson made a similar conclusion in a review of the literature that in terms of CV disease, stating that there is no good reason to recommend a switch to decaffeinated coffee.[64]

In a Japanese study of 4,587 self-defense officials with an age range of 48-56 years, Miyake et al studied the association of instant coffee and brewed coffee to serum lipids and lipoproteins.[65] After adjusting for various life-associated parameters, the authors found no relationship whatsoever of any serum lipid or lipoprotein to brewed coffee ingestion. On the other hand, the use of instant coffee had a very significant association with an increase in LDL-C whereas there was an associated decrease in triglycerides. They found that per cup of instant coffee each day, the LDL-C increased $0.82 \mathrm{mg} / \mathrm{dL}$ (95\% CI 0.29-1.35).[65] A possible explanation is that instant coffee is not filtered.

\section{Coronary heart disease (CHD)}

In a review of coffee consumption and $\mathrm{CHD}$, Gensini and Conti concluded that large prospective studies fail to support that a moderate consumption of caffeine, which they classified as <5 cups of coffee per day, significantly increases CHD risk.[66] They also determined that higher coffee intake data was so scanty that no definitive conclusion could be made despite some suggestion of a statistically significant association of CHD risk with the higher coffee intake.[66] Nawrot et al. investigated PubMed and Web of Science databases in a search for various triggers of nonfatal MI, resulting in 36 epidemiologic studies they considered of relevance to calculating a population-attributable fraction (PAF).[67] They found that the highest PAF trigger of a nonfatal MI was traffic exposure (7.4\%). Other assessed triggers included physical exertion $(6.2 \%)$, anger $(3.1 \%)$, heavy food ingestion $(2.7 \%)$, favorable emotions $(2.4 \%)$, and sexual activity $(2.2 \%)$. Coffee consumption as a trigger in their analysis was noted to be at an upper mid-level (5.0\%). Their interpretation was that prevalent small risks could possibly have important public health significance.[67] However "coffee consumption" was simply analyzed as "drinking coffee" and the relevance appears questionable but is mentioned in this article in an attempt to fairly consider all issues related to coffee intake. In an analysis of 11 prospective 
studies published between 1966 and 1991, Myers and Basinski found no relationship of coffee consumption to the development of CHD and the conclusion they made appears to remain valid in the absence of any adjustment for other CV risk factors.[68] In a meta-analysis performed by Greenland on 22 studies of CHD death, MI, and coffee consumption, it was found that coffee drinkers of 5 or more cups per day had geometric mean rate ratios of association ranging from 0.92 to 1.42 with it unlikely for there to be a ratio over 1.5 for 5 daily cups of coffee although a stronger relationship for consumption for $10 \mathrm{cups} /$ day could not be excluded.[69] Greenland concluded that the evidence for an association of coffee with CHD events remained unclear.[69] Kawachi et al. analyzed 15 cohort studies and concluded that the available data indicated that any increase in CHD risk with regular coffee consumption was very minimal.[70] Sofi et al. also concluded from their study of 13 case-control studies and 10 cohort studies that prospective cohort studies with prolonged follow up, do not show a significant relationship between CHD and regular coffee drinking in spite of some reports of a significant relationship between high coffee intake and CHD from case-control studies.[71] In support, Wu et al. performed a metaanalysis of 21 prospective cohort studies and reached the conclusion from their analysis that coffee does not increase CHD over a long period of time and they actually found that a moderate intake of coffee in women had an association with decreased CHD risk.[72] In conclusion, regarding coffee risk for CHD, at worst it appears very minimal (more likely neutral), and not a major risk factor. The assessment of Silletta and Marchioli supports this conclusion and points out the many analytical problems that include: insufficient adjustment for life-associated variables, the multiple active compounds in coffee with the potential for CV harm or benefit, the variable tolerance of the effects of caffeine with regular intake of coffee, cup size variability, methods of preparation, and the impracticality of randomized studies.[73] The appropriate clinical recommendation should be that regarding CHD, the patient should enjoy his or her coffee in moderation unless there is a direct association with bothersome symptoms.

\section{Peripheral arterial disease $(P A D)$}

The association of PAD and CHD is high, especially when PAD is severe, so the presence of an effect of coffee on PAD has to be assessed also when evaluating any relationship to $\mathrm{CV}$ disease. Uemura et al, in a study of 540 Japanese men, assessed the effect of coffee on the ankle-brachial index (ABI).[74] After making adjustments for various interfering factors including LDL-C and HDL-C, it was found that increased coffee consumption has a minimal but significant $(\mathrm{P}=0.031)$ reverse association with $\mathrm{ABI}$. This association of decreased ABI with coffee was not present with the ingestion of green tea.

\section{Congestive heart failure $(\mathrm{CHF})$}

Mostofsky et al. performed a dose-response meta-analysis of a regular consumption of coffee and the risk of congestive heart failure (CHF).[75] Their meta-analysis included a total of 5 prospective studies of coffee intake and the risk of CHF, which included 140,220 participants and a total of 6,522 episodes of CHF. The analysis of these authors resulted in a J-shaped 
relationship in which 4 cups of coffee per day had a significant inverse association with $\mathrm{CHF}$, as compared to no coffee intake. On the other hand, there appeared to be an increased risk of CHF at a coffee intake $>4$ cups per day.[75]

\section{Hypertension}

As a major CV risk factor, hypertension must be looked at in terms of any relationship to the consumption of coffee. Sudano et al. commented that the seventh report of the Joint National Committee on Prevention, Detection, Evaluation, and Treatment of High Blood Pressure (JNC 7) did not consider limitation of coffee or other beverages containing caffeine, to have an indication and that this was unnecessary.[76] They commented on the more recent state of knowledge that regular, moderate coffee ingestion is not a health hazard and of the current possible association with CV risk benefit. However, Mesas et al. do note from a review of 5 clinical trials, that in already hypertensive patients, the consumption of caffeine in a dose of 200-300 $\mathrm{mg}$ can result in an increase in systolic BP of $8.1 \mathrm{mmHg}$ and an increase in diastolic BP of $5.7 \mathrm{mmHg}$ for $\geq 3$ h.[77] Nevertheless, these authors acknowledged that up-to-date evidence does not associate coffee intake over the long term with increased BP. Other studies on the consumption of coffee and its relationship to BP and hypertension confirm that there is no statistically significant effect of coffee drinking on an increase in BP or on the development of hypertension.[78, 79] Therefore, there appears to be no BP indication for recommending against moderate coffee consumption in such patients.

\section{Plasma lipids, metabolic syndrome (MetS) and inflammation}

In a review of the effect of coffee on CV risk, Rebello and van Dam commented that the consumption of coffee is not associated with increased risk of CHD or fatal CV events and that there may be a weak association with decreased stroke and CHF risk.[80] Their review also found that unfiltered coffee result in an increase in LDL-cholesterol (LDL-C) and in triglycerides, whereas filtered coffee had no significant effect on plasma lipids. Bøhn et al. also commented that boiled coffee can contribute to an increased serum cholesterol. [81] In a rat model of human MetS, Panchal et al. found that a Colombian coffee extract given to rats fed a high-carbohydrate and high-fat diet to produce aspects of the MetS resulted in decreased glucose tolerance impairment, reduced hypertension, no change in abdominal obesity, and no change in any dyslipidemia.[82] Yesil and Yilmaz reviewed literature on the relation of consumption of coffee to metabolic syndrome and non-alcoholic fatty liver disease (NAFLD) and found that in 4 of 6 studies, there was an inverse association of coffee intake with risk for MetS, whereas the other 2 studies, without the protective effect, involved studies of young persons with low MetS prevalence.[83] In terms of NAFLD, all of the studies assessed by these authors showed a protective effect from consumption of coffee. In an assessment of endothelial function by FMD on the island of Ikaria, Greece, Siasos et al. reported a linear increase in FMD related to higher coffee consumption.[84] Actually, a boiled Greek-style coffee showed a more significant increase in FMD when compared to other kinds of coffee drinks. Corrêa et al. studied results of 2 
different types of coffee preparation on CV risk in 20 volunteers in good health, who consumed 3-4 cups of coffee per day.[85] The 2 preparations were medium light roast and medium roast, both paper-filtered. Medium light roast increased total cholesterol by $10 \%$, LDL-C by $12 \%$, and soluble vascular cell adhesion molecule-1 (sVCAM-1) by $18 \%$, whereas medium roast increased total cholesterol 12\%, LDL-C 14\%, and sVCAM-1 14\%.[85] These authors interpreted their data as showing that the moderate intake of paper-filtered coffee may result in unfavorable effects on cholesterol and markers of inflammation. In a study of a total of 364 Japanese men, Mure et al. studied the effect of coffee drinking in relationship with the MetS risk score.[86] These authors observed a favorable tendency for coffee consumption to result in decreased biological markers of the MetS and to result in a favorable increase in adiponectin. On the other hand, increased visceral fat frequently associated with the MetS results in an unfavorable decrease in adiponectin. Related to these metabolic considerations is the antioxidant status of coffee. Richelle et al studied this and found that coffee in soluble form has an antioxidant activity that is significant, is related to coffee intake, and that the antioxidant effect of the coffee has a long lag time of 292-948 min.[87] Interestingly, Richelle et al. found some differences in the type of coffee beans; green Robusta coffee beans had twice the antioxidant effect as did Arabica coffee beans, the difference of which was not significant after roasting.[87] In addition, it appears important that the antioxidant effect of coffee have a long lag time; Carru et al., in an in vitro copper sulphate-catalyzed study of LDL oxidation, found that the antioxidant effect of coffee ultimately switched to pro-oxidant when there was a significant prolonged lag time.[88]

\section{Stroke}

Any association of coffee with the occurrence of stroke needs to be considered on its own. The best available information appears to have been found in observational meta-analyses and there has been an inconsistent association of coffee intake with stroke risk. However, in a meta-analysis of 11 prospective studies, including 10,003 stroke patients from 479,689 individuals, Larsson and Orsini found some evidence of an association of coffee intake and stroke risk that was nonlinear.[89] They found RRs as follows: 2 cups per day: RR 00.86; 3-4 cups per day: RR 0.83; 6 cups per day: RR 0.87; and for 8 cups per day, RR 0.93. These authors concluded that moderate coffee intake may have a weak inverse association with stroke risk. In another meta-analysis, Kim et al. looked at 9 studies regarding stroke risk and the use of coffee and found that increased coffee consumption had a preventive advantage by decreasing stroke incidence. Those drinking 4 or more cups of coffee per day had RR 0.83 (95\% CI, 0.750.91).[90] In contrast, a combined analysis of sugar-sweetened soda intake from the Nurses' Health Study and the Health Professionals Follow-Up Study showed a pooled RR 1.16 (95\% CI, 1.00-1.34) for total stroke incidence compared to one cup caffeinated coffee per day, with a $9 \%$ decreased stroke risk (95\% CI, 0-17\%), and one cup decaffeinated coffee per day with $10 \%$ decreased stroke risk (95\% CI, 1-19\%).[91] In a recent article, Larsson assessed that available prospective studies have found in general a weak inverse association between moderate coffee intake and stroke risk.[92] On the other hand, Lee et al performed a case-control study of 940 
patients with acute hemorrhagic stroke not involving head injury, and found that using medications containing caffeine was associated with increased risk of hemorrhagic stroke, including both subarachnoid and intracerebral hemorrhage.[93] Analysis of available data therefore supports coffee and its myriad other substances and not caffeine as offering some protection from stroke.

\section{Mortality}

The relationship of coffee to mortality is another issue for consideration that was addressed by Freedman et al. in an extensive analysis of total cause-specific mortality involving 229,119 men and 173,141 women, aged 50-71 years as a prospective study.[94] They found that the consumption of coffee was inversely associated with total and cause-specific mortality but considered that their data could not define whether coffee was just an association vs. having status as a causative factor. In another meta-analysis, Je and Giovannucci performed a metaanalysis on 129,538 deaths involving 973,904 participants from 20 studies.[95] Their analysis showed an RR for total mortality for high vs. low coffee intake to be 0.86 (95\% CI, 0.80-0.92), and for those studies defining heavy coffee use as $\geq 2-4$ cups per day or heavy intake as $\geq 5-9$ cups per day, there was a significant difference and they were similar. This favorable inverse association had a stronger tendency in European and Japanese studies than for the United States where the risk favorable inverse association with coffee consumption was less. The favorable inverse association was present for both men and women and their ultimate interpretation was that the consumption of coffee is related to decreased mortality risk.[95] Malerba et al. also performed a review and meta-analysis on the association of coffee with all-cause mortality and CV mortality.[96] They reported that their quantitative evidence supported the fact that the consumption of coffee decreases all-cause mortality and probably CV disease mortality. On the other hand, Liu et al. analyzed data from the Aerobics Center Longitudinal Study with 43,727 participants.[97] In this large group, these authors found that heavy coffee intake, defined as more than 28 cups per week, had a positive correlation with all-cause mortality in both men and women who were less than 55 years of age. Therefore, Liu et al. stated that it appeared indicated to suggest that individuals at a younger age should avoid such heavy coffee drinking.[97] However, in a subsequent follow up, Liu et al. noted that it may be premature to render advice on a limitation of coffee drinking.[98]

\section{Specific vascular effects of coffee}

There are multiple possible vascular effects of coffee, a comprehensive discussion of which is beyond the scope of this selected overview. However, it appears appropriate to summarize some of the possible considerations. Schechter et al. looked at essentially the immediate effect of caffeine ingestion on endothelial function as assessed by brachial artery FMD.[99] There were 40 controls free of known CHD and 40 matched participants with known CHD. Participants from both groups were given either placebo or a $200 \mathrm{mg}$ caffeine capsule while in a fasting state and with no caffeine in excess of $48 \mathrm{~h}$. In participants, both with and 
without $\mathrm{CHD}$, it was observed that $1 \mathrm{~h}$ after caffeine consumption, there was a significant improvement in endothelial function as evaluated by brachial artery FMD and decreased highsensitivity C-reactive protein as a marker of reduced inflammation,[99] both representing potential benefit to the CV system. Reis et al, in the Coronary Artery Risk Development in Young Adults (CARDIA) Study, assessed the effect of coffee and caffeine on carotid artery intima-media thickness and on coronary artery calcium score out to 20 years of follow up.[100] These authors found no significant association between coffee or caffeine on either measure of vascular atherosclerosis,[100] consistent with at least no harmful effects. Catalano et al. studied the effect of coffee on 137 patients with non-alcoholic fatty liver disease (NAFLD) and 108 controls.[101] They found that coffee consumption was associated with decreased NAFLD and decreased insulin resistance,[101] both of potential metabolic and vascular benefit. NAFLD is associated with metabolic syndrome and increased vascular risk.[102] In a study on the cremasteric arteries of mice, Toda et al. found that the administration of coffee appeared to have a beneficial effect in preventing thrombus development in arteries whose endothelial function had been unfavorably altered by the administration of ferric chloride.[103] These authors did not find this beneficial effect with caffeine administration alone and their study supports further interest in specific non-caffeine coffee effects on the vasculature.[103] The studies just discussed are a sample of the extensive reported information to be considered regarding the effects of coffee at the vascular level, a full consideration of which is not possible in this overview (or in any single overview).

\section{Conclusions}

The status of coffee as offering benefit in decreasing $\mathrm{CV}$ risk remains unresolved from a hard, universally-accepted numbers standpoint. Nevertheless, there is favorable evidence that, unless there is a specific high-risk genetic abnormality, coffee consumption has supportive evidence for a reduction in all-cause mortality and a reduction in $\mathrm{CV}$ mortality. The data is much stronger in support of a decreased incidence of type 2 DM with an established and accepted benefit of coffee consumption in regard to this disease. The complex chemistry of coffee needs much study and understanding such as the apparent higher CV risk associated with unfiltered boiled coffee and the potentially harmful plasma lipid-elevating components that may be removed by filtration. In general, appropriate advice by the clinician to his or her patients is that coffee is to be enjoyed by them, even when they are in the highest category of CV risk, if they like the beverage. Only if coffee is associated with bothersome symptomatic CV manifestations such as arrhythmias, should limitation or cessation be suggested.

\section{Acknowledgement}

The author wishes to recognize the excellent editorial critique of Susan Quick and its contribution to this manuscript. 


\section{Declaration of interest}

The author has no conflicts of interest to declare with any pharmaceutical or medical device company. Also, he has no stock ownership or other ownership conflict to report.

\section{REFERENCES}

[1] The History of Coffee. National Coffee Association of U.S.A.; 2009 [March 30, 2014]; Available from: http://www.ncausa.org/i4a/pages/index.cfm?pageid=68.

[2] Whayne T, Jr. Una taza de café y otras terapias alternativas en medicina clínica. Rev Costarr Cardiol 2009;11:43-48.

[3] Ludwig IA, Clifford MN, Lean ME, et al. Coffee: biochemistry and potential impact on health. Food Funct 2014.

[4] Bonita JS, Mandarano M, Shuta D, Vinson J. Coffee and cardiovascular disease: in vitro, cellular, animal, and human studies. Pharmacol Res 2007;55:187-98.

[5] Park JB. 5-Caffeoylquinic acid and caffeic acid orally administered suppress P-selectin expression on mouse platelets. J Nutr Biochem 2009;20:800-5.

[6] Poquet L, Clifford MN, Williamson G. Effect of dihydrocaffeic acid on UV irradiation of human keratinocyte HaCaT cells. Arch Biochem Biophys 2008;476:196-204.

[7] Suzuki A, Kagawa D, Ochiai R, et al. Green coffee bean extract and its metabolites have a hypotensive effect in spontaneously hypertensive rats. Hypertens Res 2002;25:99-107.

[8] Lee C. Antioxidant ability of caffeine and its metabolites based on the study of oxygen radical absorbing capacity and inhibition of LDL peroxidation. Clin Chim Acta 2000;295:14154.

[9] Halvorsen BL, Carlsen MH, Phillips KM, et al. Content of redox-active compounds (ie, antioxidants) in foods consumed in the United States. Am J Clin Nutr 2006;84:95-135.

[10] Cornelis MC, El-Sohemy A, Kabagambe EK, Campos H. Coffee, CYP1A2 genotype, and risk of myocardial infarction. JAMA 2006;295:1135-41.

[11] Sulem P, Gudbjartsson DF, Geller F, et al. Sequence variants at CYP1A1-CYP1A2 and AHR associate with coffee consumption. Hum Mol Genet 2011;20:2071-7.

[12] Amin N, Byrne E, Johnson J, et al. Genome-wide association analysis of coffee drinking suggests association with CYP1A1/CYP1A2 and NRCAM. Mol Psychiatry 2012;17:1116-29.

[13] Bertoia ML, Triche EW, Michaud DS, et al. Long-term alcohol and caffeine intake and risk of sudden cardiac death in women. Am J Clin Nutr 2013;97:1356-63.

[14] Park JJ, Grubb N. Solving the puzzle of strong French coffee, palpitation and dizziness. BMJ Case Rep 2013;2013.

[15] Caldeira D, Martins C, Alves LB, et al. Caffeine does not increase the risk of atrial fibrillation: a systematic review and meta-analysis of observational studies. Heart 2013;99:13839.

[16] Bhave PD, Hoffmayer K. Caffeine and atrial fibrillation: friends or foes? Heart 2013;99:1377-8.

[17] van Dam RM. Coffee consumption and risk of type 2 diabetes, cardiovascular diseases, and cancer. Appl Physiol Nutr Metab 2008;33:1269-83. 
[18] Muley A, Muley P, Shah M. Coffee to reduce risk of type 2 diabetes?: a systematic review. Curr Diabetes Rev 2012;8:162-8.

[19] Jiang X, Zhang D, Jiang W. Coffee and caffeine intake and incidence of type 2 diabetes mellitus: a meta-analysis of prospective studies. Eur J Nutr 2014;53:25-38.

[20] Ding M, Bhupathiraju SN, Chen M, et al. Caffeinated and decaffeinated coffee consumption and risk of type 2 diabetes: a systematic review and a dose-response meta-analysis. Diabetes Care 2014;37:569-86.

[21] Bhupathiraju SN, Pan A, Malik VS, et al. Caffeinated and caffeine-free beverages and risk of type 2 diabetes. Am J Clin Nutr 2013;97:155-66.

[22] Natella F, Scaccini C. Role of coffee in modulation of diabetes risk. Nutr Rev 2012;70:207-17.

[23] Wierzejska R. [Caffeine--common ingredient in a diet and its influence on human health]. Rocz Panstw Zakl Hig 2012;63:141-7.

[24] Barone JJ, Roberts HR. Caffeine consumption. Food Chem Toxicol 1996;34:119-29.

[25] Market: Coffee consumption statistics in the United States.

[26] Ding M, Bhupathiraju SN, Satija A, et al. Long-term coffee consumption and risk of cardiovascular disease: a systematic review and a dose-response meta-analysis of prospective cohort studies. Circulation 2014;129:643-59.

[27] O'Keefe JH, Bhatti SK, Patil HR, et al. Effects of habitual coffee consumption on cardiometabolic disease, cardiovascular health, and all-cause mortality. J Am Coll Cardiol 2013;62:1043-51.

[28] Patil H, Lavie CJ, O'Keefe JH. Cuppa joe: friend or foe? Effects of chronic coffee consumption on cardiovascular and brain health. Mo Med 2011;108:431-8.

[29] Floegel A, Pischon T, Bergmann MM, et al. Coffee consumption and risk of chronic disease in the European Prospective Investigation into Cancer and Nutrition (EPIC)-Germany study. Am J Clin Nutr 2012;95:901-8.

[30] Montagnana M, Favaloro EJ, Lippi G. Coffee intake and cardiovascular disease: virtue does not take center stage. Semin Thromb Hemost 2012;38:164-77.

[31] Lopez-Garcia E. Coffee consumption and risk of chronic diseases: changing our views. Am J Clin Nutr 2012;95:787-8.

[32] Ahola I, Jauhiainen M, Aro A. The hypercholesterolaemic factor in boiled coffee is retained by a paper filter. J Intern Med 1991;230:293-7.

[33] van Dusseldorp M, Katan MB, van Vliet T, et al. Cholesterol-raising factor from boiled coffee does not pass a paper filter. Arterioscler Thromb 1991;11:586-93.

[34] Pirich C, O'Grady J, Sinzinger H. Coffee, lipoproteins and cardiovascular disease. Wien Klin Wochenschr 1993;105:3-6.

[35] Bak AA, Grobbee DE. The effect on serum cholesterol levels of coffee brewed by filtering or boiling. N Engl J Med 1989;321:1432-7.

[36] Aro A, Teirila J, Gref CG. Dose-dependent effect on serum cholesterol and apoprotein B concentrations by consumption of boiled, non-filtered coffee. Atherosclerosis 1990;83:257-61.

[37] Aro A, Tuomilehto J, Kostiainen E, et al. Boiled coffee increases serum low density lipoprotein concentration. Metabolism 1987;36:1027-30.

[38] Rosmarin PC, Applegate WB, Somes GW. Coffee consumption and serum lipids: a randomized, crossover clinical trial. Am J Med 1990;88:349-56. 
[39] Green MS, Harari G. Association of serum lipoproteins and health-related habits with coffee and tea consumption in free-living subjects examined in the Israeli CORDIS Study. Prev Med 1992;21:532-45.

[40] Fried RE, Levine DM, Kwiterovich PO, et al. The effect of filtered-coffee consumption on plasma lipid levels. Results of a randomized clinical trial. JAMA 1992;267:811-5.

[41] Salvaggio A, Periti M, Miano L, et al. Coffee and cholesterol, an Italian study. Am J Epidemiol 1991;134:149-56.

[42] D'Amicis A, Scaccini C, Tomassi G, et al. Italian style brewed coffee: effect on serum cholesterol in young men. Int J Epidemiol 1996;25:513-20.

[43] van Tol A, Urgert R, de Jong-Caesar R, et al. The cholesterol-raising diterpenes from coffee beans increase serum lipid transfer protein activity levels in humans. Atherosclerosis 1997;132:251-4.

[44] Urgert R, Essed N, van der Weg G, et al. Separate effects of the coffee diterpenes cafestol and kahweol on serum lipids and liver aminotransferases. Am J Clin Nutr 1997;65:519-24.

[45] De Roos B, Van Tol A, Urgert R, et al. Consumption of French-press coffee raises cholesteryl ester transfer protein activity levels before LDL cholesterol in normolipidaemic subjects. J Intern Med 2000;248:211-6.

[46] Rustan AC, Halvorsen B, Huggett AC, et al. Effect of coffee lipids (cafestol and kahweol) on regulation of cholesterol metabolism in HepG2 cells. Arterioscler Thromb Vasc Biol 1997; 17:2140-9.

[47] Natella F, Nardini M, Belelli F, Scaccini C. Coffee drinking induces incorporation of phenolic acids into LDL and increases the resistance of LDL to ex vivo oxidation in humans. Am J Clin Nutr 2007;86:604-9.

[48] Kishimoto Y, Tani M, Kondo K. Pleiotropic preventive effects of dietary polyphenols in cardiovascular diseases. Eur J Clin Nutr 2013;67:532-5.

[49] Williams PT, Wood PD, Vranizan KM, et al. Coffee intake and elevated cholesterol and apolipoprotein B levels in men. JAMA 1985;253:1407-11.

[50] Aro A, Kostiainen E, Huttunen JK, et al. Effects of coffee and tea on lipoproteins and prostanoids. Atherosclerosis 1985;57:123-8.

[51] Donahue RP, Orchard TJ, Stein EA, Kuller LH. Lack of an association between coffee consumption and lipoprotein lipids and apolipoproteins in young adults: the Beaver County Study. Prev Med 1987;16:796-802.

[52] Paoletti R, Corsini A, Tremoli E, et al. Effects of coffee on plasma lipids, lipoproteins and apolipoproteins. Pharmacol Res 1989;21:27-38.

[53] Wilson PW, Garrison RJ, Kannel WB, et al. Is coffee consumption a contributor to cardiovascular disease? Insights from the Framingham Study. Arch Intern Med 1989;149:116972 .

[54] Kohlmeier L, Mensink G, Kohlmeier M. The relationship between coffee consumption and lipid levels in young and older people in the Heidelberg-Michelstadt-Berlin study. Eur Heart J 1991;12:869-74.

[55] Carson CA, Cauley JA, Caggiula AW. Relation of caffeine intake to blood lipids in elderly women. Am J Epidemiol 1993;138:94-100.

[56] Jee SH, He J, Appel LJ, et al. Coffee consumption and serum lipids: a meta-analysis of randomized controlled clinical trials. Am J Epidemiol 2001;153:353-62.

[57] Kokjohn K, Graham M, McGregor M. The effect of coffee consumption on serum cholesterol levels. J Manipulative Physiol Ther 1993;16:327-35. 
[58] Cai L, Ma D, Zhang Y, et al. The effect of coffee consumption on serum lipids: a metaanalysis of randomized controlled trials. Eur J Clin Nutr 2012;66:872-7.

[59] Haffner SM, Knapp JA, Stern MP, et al. Coffee consumption, diet, and lipids. Am J Epidemiol 1985;122:1-12.

[60] Cheung RJ, Gupta EK, Ito MK. Acute coffee ingestion does not affect LDL cholesterol level. Ann Pharmacother 2005;39:1209-13.

[61] Jossa F, Krogh V, Farinaro E, et al. Coffee and serum lipids: findings from the Olivetti Heart Study. Ann Epidemiol 1993;3:250-5.

[62] Superko HR, Bortz W, Jr., Williams PT, et al. Caffeinated and decaffeinated coffee effects on plasma lipoprotein cholesterol, apolipoproteins, and lipase activity: a controlled, randomized trial. Am J Clin Nutr 1991;54:599-605.

[63] van Dusseldorp M, Katan MB, Demacker PN. Effect of decaffeinated versus regular coffee on serum lipoproteins. A 12-week double-blind trial. Am J Epidemiol 1990;132:33-40.

[64] Thompson WG. Coffee: brew or bane? Am J Med Sci 1994;308:49-57.

[65] Miyake Y, Kono S, Nishiwaki M, et al. Relationship of coffee consumption with serum lipids and lipoproteins in Japanese men. Ann Epidemiol 1999;9:121-6.

[66] Gensini GF, Conti AA. [Does coffee consumption represent a coronary risk factor?]. Recenti Prog Med 2004;95:563-5.

[67] Nawrot TS, Perez L, Kunzli N, et al. Public health importance of triggers of myocardial infarction: a comparative risk assessment. Lancet 2011;377:732-40.

[68] Myers MG, Basinski A. Coffee and coronary heart disease. Arch Intern Med 1992;152:1767-72.

[69] Greenland S. A meta-analysis of coffee, myocardial infarction, and coronary death. Epidemiology 1993;4:366-74.

[70] Kawachi I, Colditz GA, Stone CB. Does coffee drinking increase the risk of coronary heart disease? Results from a meta-analysis. Br Heart J 1994;72:269-75.

[71] Sofi F, Conti AA, Gori AM, et al. Coffee consumption and risk of coronary heart disease: a meta-analysis. Nutr Metab Cardiovasc Dis 2007;17:209-23.

[72] Wu JN, Ho SC, Zhou C, et al. Coffee consumption and risk of coronary heart diseases: a meta-analysis of 21 prospective cohort studies. Int J Cardiol 2009;137:216-25.

[73] Silletta MG, Marchioli R. [Coffee and cardiovascular disease risk: yin and yang]. Recenti Prog Med 2008;99:533-7.

[74] Uemura H, Katsuura-Kamano S, Yamaguchi M, et al. Consumption of coffee, not green tea, is inversely associated with arterial stiffness in Japanese men. Eur J Clin Nutr 2013;67:110914.

[75] Mostofsky E, Rice MS, Levitan EB, Mittleman MA. Habitual coffee consumption and risk of heart failure: a dose-response meta-analysis. Circ Heart Fail 2012;5:401-5.

[76] Sudano I, Binggeli C, Spieker L, et al. Cardiovascular effects of coffee: is it a risk factor? Prog Cardiovasc Nurs 2005;20:65-9.

[77] Mesas AE, Leon-Munoz LM, Rodriguez-Artalejo F, Lopez-Garcia E. The effect of coffee on blood pressure and cardiovascular disease in hypertensive individuals: a systematic review and meta-analysis. Am J Clin Nutr 2011;94:1113-26.

[78] Steffen M, Kuhle C, Hensrud D, et al. The effect of coffee consumption on blood pressure and the development of hypertension: a systematic review and meta-analysis. $\mathrm{J}$ Hypertens 2012;30:2245-54. 
[79] Zhang Z, Hu G, Caballero B, et al. Habitual coffee consumption and risk of hypertension: a systematic review and meta-analysis of prospective observational studies. Am J Clin Nutr 2011;93:1212-9.

[80] Rebello SA, van Dam RM. Coffee consumption and cardiovascular health: getting to the heart of the matter. Curr Cardiol Rep 2013;15:403.

[81] Bohn SK, Ward NC, Hodgson JM, Croft KD. Effects of tea and coffee on cardiovascular disease risk. Food Funct 2012;3:575-91.

[82] Panchal SK, Poudyal H, Waanders J, Brown L. Coffee extract attenuates changes in cardiovascular and hepatic structure and function without decreasing obesity in highcarbohydrate, high-fat diet-fed male rats. J Nutr 2012;142:690-7.

[83] Yesil A, Yilmaz Y. Review article: coffee consumption, the metabolic syndrome and non-alcoholic fatty liver disease. Aliment Pharmacol Ther 2013;38:1038-44.

[84] Siasos G, Oikonomou E, Chrysohoou C, et al. Consumption of a boiled Greek type of coffee is associated with improved endothelial function: the Ikaria study. Vasc Med 2013;18:5562.

[85] Correa TA, Rogero MM, Mioto BM, et al. Paper-filtered coffee increases cholesterol and inflammation biomarkers independent of roasting degree: a clinical trial. Nutrition 2013;29:97781.

[86] Mure K, Maeda S, Mukoubayashi C, et al. Habitual coffee consumption inversely associated with metabolic syndrome-related biomarkers involving adiponectin. Nutrition 2013;29:982-7.

[87] Richelle M, Tavazzi I, Offord E. Comparison of the antioxidant activity of commonly consumed polyphenolic beverages (coffee, cocoa, and tea) prepared per cup serving. J Agric Food Chem 2001;49:3438-42.

[88] Carru C, Pasciu V, Sotgia S, et al. The Oxidative State of LDL is the Major Determinant of Anti/Prooxidant Effect of Coffee on Cu Catalysed Peroxidation. Open Biochem J 2011;5:1-8. [89] Larsson SC, Orsini N. Coffee consumption and risk of stroke: a dose-response metaanalysis of prospective studies. Am J Epidemiol 2011;174:993-1001.

[90] Kim B, Nam Y, Kim J, et al. Coffee Consumption and Stroke Risk: A Meta-analysis of Epidemiologic Studies. Korean J Fam Med 2012;33:356-65.

[91] Bernstein AM, de Koning L, Flint AJ, et al. Soda consumption and the risk of stroke in men and women. Am J Clin Nutr 2012;95:1190-9.

[92] Larsson SC. Coffee, tea, and cocoa and risk of stroke. Stroke 2014;45:309-14.

[93] Lee SM, Choi NK, Lee BC, et al. Caffeine-containing medicines increase the risk of hemorrhagic stroke. Stroke 2013;44:2139-43.

[94] Freedman ND, Park Y, Abnet CC, et al. Association of coffee drinking with total and cause-specific mortality. N Engl J Med 2012;366:1891-904.

[95] Je Y, Giovannucci E. Coffee consumption and total mortality: a meta-analysis of twenty prospective cohort studies. Br J Nutr 2014;111:1162-73.

[96] Malerba S, Turati F, Galeone C, et al. A meta-analysis of prospective studies of coffee consumption and mortality for all causes, cancers and cardiovascular diseases. Eur J Epidemiol 2013;28:527-39.

[97] Liu J, Sui X, Lavie CJ, et al. Association of coffee consumption with all-cause and cardiovascular disease mortality. Mayo Clin Proc 2013;88:1066-74.

[98] Liu J, Sui X, Blair SN, Lavie CJ. In reply--Association of coffee consumption with allcause and cardiovascular disease mortality. Mayo Clin Proc 2013;88:1493-4. 
[99] Shechter M, Shalmon G, Scheinowitz M, et al. Impact of acute caffeine ingestion on endothelial function in subjects with and without coronary artery disease. Am J Cardiol 2011;107:1255-61.

[100] Reis JP, Loria CM, Steffen LM, et al. Coffee, decaffeinated coffee, caffeine, and tea consumption in young adulthood and atherosclerosis later in life: the CARDIA study.

Arterioscler Thromb Vasc Biol 2010;30:2059-66.

[101] Catalano D, Martines GF, Tonzuso A, et al. Protective role of coffee in non-alcoholic fatty liver disease (NAFLD). Dig Dis Sci 2010;55:3200-6.

[102] Athyros VG, Katsiki N, Karagiannis A, Mikhailidis DP. Are statins 'IDEAL' for nonalcoholic fatty liver disease? Curr Med Res Opin 2014;30:229-31.

[103] Toda E, Ishida H, Aoki T, et al. Possible mechanism of preventive effects of coffee intake on the formation of arterial occlusive thrombosis. Tokai J Exp Clin Med 2010;35:133-6.

Table 1: Summary of the beneficial and harmful effects of coffee.

\begin{tabular}{|c|c|c|c|}
\hline Probable benefit & Definite benefit & Probable harm & Definite harm \\
\hline $\begin{array}{l}\text { Possible decreased cardio- } \\
\text { vascular (CV) risk due to } \\
\text { weight reduction, increased } \\
\text { fibrinolysis, and increased } \\
\text { arterial flow-mediated dilata- } \\
\text { tion (FMD).[30] }\end{array}$ & $\begin{array}{l}\text { Decreased onset of type } 2 \\
\text { diabetes mellitus } \\
\text { (DM).[18-20] This } \\
\text { appears well established. }\end{array}$ & $\begin{array}{l}\text { Increased congestive heart } \\
\text { failure }(\mathrm{CHF}) \text { with coffee intake } \\
\text { over } 4 \text { cups per day.[75] }\end{array}$ & $\begin{array}{l}\text { Increased nonfatal } \\
\text { myocardial infarction } \\
\text { (MI) in the presence of } \\
\text { slow caffeine } \\
\text { metabolism.[15] }\end{array}$ \\
\hline $\begin{array}{l}\text { Some suggestion of } \\
\text { decreased overall stroke } \\
\text { risk.[89-91] }\end{array}$ & & $\begin{array}{l}\text { Variable association of coffee } \\
\text { with mild increases in plasma } \\
\text { cholesterol, low-density } \\
\text { lipoprotein cholesterol (LDL- } \\
\text { C), and triglycerides.[58, } 81 \text {, } \\
\text { 85] }\end{array}$ & \\
\hline $\begin{array}{l}\text { General decrease in all-cause } \\
\text { mortality risk.[94-96] }\end{array}$ & & $\begin{array}{l}\text { Increased all-cause mortality } \\
\text { under age } 55 \text { years with heavy } \\
\text { coffee intake.[97] }\end{array}$ & \\
\hline
\end{tabular}

